\title{
CIRP Promotes the Progression of Non-small Cell Lung Cancer Through Activation of Wnt/ $\beta$-catenin Signaling via CTNNB1
}

Yi Liao

Army Medical University

Jianguo Feng

Southwest Medical University

Weichao Sun

Shenzhen Second People's Hospital

Chao Wu

Army Medical University

Jingyao Li

Army Medical University

Tao Jing

Army Medical University

Yuteng Liang

Shenzhen Second People's Hospital

Yonghui Qian

Shenzhen Second People's Hospital

Wenlan Liu

Shenzhen Second People's Hospital

Haidong Wang ( $\nabla$ haidongwang1970@163.com)

Third Military Medical University https://orcid.org/0000-0003-4409-4339

Research

Keywords: Non-small cell lung cancer, CIRP, CTNNB1, Wnt/ $\beta$-Catenin

Posted Date: April 13th, 2021

DOI: https://doi.org/10.21203/rs.3.rs-358107/v1

License: (1) (1) This work is licensed under a Creative Commons Attribution 4.0 International License.

Read Full License 


\section{Abstract}

Background: Cold-inducible RNA binding protein (CIRP) is a newly discovered proto-oncogene. In this study, we investigated the role of CIRP in the progression of non-small cell lung cancer (NSCLC) using clinic samples, cultured cell lines and animal lung cancer models.

Methods: Tissue arrays, IHC and HE staining, immunoblotting, and qRT-PCR were used to detect the indicated gene expression; Plasmid and siRNA transfections as well as viral infection were used to manipulate gene expression; Cell proliferation assay, cell cycle analysis, cell migration and invasion analysis, soft agar colony formation assay, tail intravenous injecting and subcutaneously inoculating of animal models were performed to study the role of CIRP in NSCLC cells; Gene expression microarray was used to select the underlying pathways; RNA immunoprecipitation assay, biotin pull-down assay, immuno-purification assay, mRNA decay analyses and luciferase reporter assay were performed to elucidate the mechanisms. The log-rank (Mantel-Cox) test, independent sample T test, the nonparametric Mann-Whitney test, spearman rank test and two-tailed independent sample T-test were used accordingly in our study.

Results: Our data showed that CIRP was highly expressed in NSCLC tissue, and its level was negatively correlated with the prognosis of NSCLC patients. By manipulating CIRP expression in A549, H460, H1299, and $\mathrm{H} 1650$ cell lines, we demonstrated that CIRP overexpression promoted the transition of G1/G0 phase to $S$ phase and the formation of enhanced malignant phenotype of NSCLC, reflected by increased proliferation, enhanced invasion/metastasis and greater tumorigenic capabilities both in vitro and in vivo. Transcriptome sequencing further demonstrated that CIRP acted on cell cycle, DNA replication and Wnt signaling pathway to exert its pro-oncogenic action. Mechanistically, CIRP directly bound to the 3'- and 5'UTR of CTNNB1 mRNA, leading to enhanced stability and translation of CTNNB1 mRNA and promote IRES-mediated protein synthesis, respectively. Eventually, the increased CTNNB1 protein levels mediated excessive activation of the Wnt/ $\beta$-Catenin signaling pathway and its downstream C-myc, COX-2, CCND1, MMP7, VEGFA and CD44.

Conclusion: Our results support CIRP as a candidate oncogene in NSCLC and a potential target for NSCLC therapy.

\section{Background}

Non-small cell lung cancer (NSCLC) is one of the most malignant tumors, with worldwide increasing incidence (1). Although the diagnostic and treatment algorithms for NSCLC are constantly improved, it is difficult to obtain satisfactory curative effect, and the long-term survival rate is still low (2). A relatively poor understanding of the molecular mechanisms underlying the pathogenesis of NSCLC is one of the reasons. A plenty of studies have been focused on identification of NSCLC associated genes (3-5). However, the molecular pathogenesis of NSCLC is far from being clear elucidated. Therefore, searching 
for new genes that are involved in development of NSCLC is critical for identifying potential therapeutic targets (6).

Cold-inducible RNA binding protein (CIRP) is constitutively expressed in the nucleus at low levels in various tissues, and its expression can be induced by mild hypothermia, UV, hypoxia, osmotic pressure, and ischemic disorders (7). CIRP contains an RNA recognition motif (RRM) domain with RNA binding function and a glycine-rich C-terminal domain involved in cell localization (8). It is believed that CIRP is a stress-induced protein and participates in multiple cellular signaling pathways by post-transcriptional regulating of the translation of specific mRNAs. Studies have shown that tumor necrosis factor-alpha (TNF-a), cycloheximide, or hypothermia treatment could upregulate the expression of CIRP and thus increase the expression of phosphorylated extracellular signal-regulated kinase 1/2 (ERK1/2), leading to the inhibition of apoptosis signal acquisition factor caspase-3 and the bypassing of the replicative senescence (9-13). In addition, CIRP could post-transcriptionally regulate the expression of tumorous bio-markers RPA2 and TRX $(14,15)$, mediating the expression of poor prognosis-related genes such as hypoxia inducible transcriptional factor-1 (HIF-1) and vascular endothelial growth factor (VEGF) (16-18). Moreover, studies have also reported that CIRP upregulation increased the expression of cell cycle-related proteins, such as cyclin D1 and c-Myc $(19,20)$. In another study, CIRP was shown to negatively regulate p53 levels, thereby down-regulating pro-apoptotic genes and up-regulating anti-apoptotic genes (21). These findings all support CIRP as a new proto-oncogene to promote the progression of various cancers through multiple cellular signal pathways, such as brain cancer, breast cancer, oral cancer, colon cancer, prostate cancer, and liver cancer (22). However, the mechanisms underlying the role(s) of CIRP in the regulation of NSCLC progression remain largely unknown.

In this study, we investigated CIRP expression and its functions in NSCLC. Our data showed that CIRP was overexpressed in NSCLC cell lines and human NSCLC tissue, and CIRP overexpression was correlated with a poor prognosis in NSCLC patients. Mechanistically, CIRP directly bound to the 3'- and 5'-UTRs of CTNNB1 mRNA to enhance its stability and translation, and the resultant accumulation of CTNNB1 protein induced excessive activation of Wnt/ $\beta$-Catenin signaling and promoted the progression of NSCLC.

\section{Materials And Methods}

\section{Tissue arrays}

Eighty-six tumor tissues and their paired noncancerous tissues were randomly collected from NSCLC patients who had undergone surgical resection in the Department of Thoracic Surgery, Southwest Hospital, Army Medical University (Chongqing, China). No antitumor treatment was performed before the surgery. All samples were frozen into liquid nitrogen within $10 \mathrm{~min}$ following surgical resection and stored at $-80^{\circ} \mathrm{C}$ until analyses. Tissue array blocks containing NSCLC tissues and adjunction noncancerous tissues were established using a tissue microarrayer (Leica, Germany). Procedures for the collection of human samples and their usage for tissue arrays were approved by the Ethical Committee of the Army 
Medical University (Chongqing, China). Informed consent forms were obtained from the patients before participating in this study.

\section{Immunohistochemistry (IHC) and Hematoxylin-Eosin (HE) staining}

The streptavidin-biotin peroxidase complex method was used for immunohistochemical staining of tissue array slides and the formalin-fixed, paraffin embedded tissue sections. Antigen retrieval was performed by heating the dewaxed and dehydrated sections in Dako antigen retrieval solution containing 10 mM EDTA ( $\mathrm{pH}$ 8.0) with a pressure cooker. Goat anti-human CIRP antibody (ab106230, Abcam, USA; 1 : 250 dilution) was used to detect CIRP expression. Rabbit anti-human CTNNB1 antibody (19807S, Cell Signaling Technology, USA; 1 : 200 dilution) was used to detect CTNNB1 expression. The expression of CIRP and CTNNB1 was evaluated using graded semi-quantitatively scoring system. The intensity of staining was classified into none (0), weak (1), strong (2) or very strong (3), and the staining patterns were classified into negative (0: $\leq 10 \%)$, sporadic (1: $11-25 \%)$, focal $(2: 26-50 \%)$ or diffuse ( 3 : $\geq 51 \%)$. An overall score was calculated by multiplying intensity and positivity scores as follows: 0 (negative), 1 and 2 (weak staining), 3 and 4 (moderately staining), 6 and 9 (strongly staining). To analyze clinical significance and prognosis, malignant samples with strongly CIRP/CTNNB1 staining were classified as high-expression, whereas low-expression indicated moderately staining, weak staining and negative staining. The paraffin-embedded tumor sections were used for examination of HE staining following standard protocols. Photos were taken using a light microscope.

\section{Cell culture}

Human lung cancer cell lines A549, NCl-H1299, NCl-H1650, NCl-H460 and NCl-H446 were purchased from the SUYANBIOTECH (Guangzhou, China). A549 cells with stable luciferase expression (A549-Luc ${ }^{+}$) were purchased from AIPONUO (Guangzhou, China). HEK293FT and HaCaT cells were obtained from Dr. Tang's Lab (Chongqing, China). All cells were identified by short tandem repeat (STR) profiling and cultured according to the manufacturer's specifications for less than 3 months. For stable selection of H1299/Le-CIRP and H1650/Le-CIRP cells, the cultural medium was supplemented with $2.0 \mu \mathrm{g} / \mathrm{mL}$ puromycin (\#P8833, Sigma-Aldrich, MO, USA).

\section{Animal experiments}

Female BALB/c nude mice (4-weeks old) were purchased from Beijing Huafukang Biosicence Co., Ltd. (Beijing, China), and maintained in the Experimental Animal Center of Army Medical University (Chongqing, China). All procedures for animal experiments were approved by the Committee on the Use and Care on Animals (Army Medical University, Chongqing, China) and performed in accordance with institution guidelines. After adaptive feeding for two weeks, pulmonary metastasis models were established by tail intravenous injecting $1 \times 10^{5}$ cells diluted in $100 \mu \mathrm{L} \mathrm{PBS}$ (A549-Luc ${ }^{+} /$Le-scrambled $^{2}$ infected group, $n=6$; A549-Luc ${ }^{+} /$Le-shCIRP infected group, $n=6$ ). The bioluminescence of pulmonary metastasis tumor was measured by The Xenogen IVIS-200 System (Xenogen, USA). The survival of mice 
after cell transplantation was recorded and analyzed accordingly. After infection with indicated lentiviral vectors, A549/H460 tumor xenografts were established by subcutaneously inoculating $1 \times 10^{6}$ cells into the 6-week-old female BALB/c nude mice (A549/Le-scrambled infected group, $n=9 ;$ A549/Le-shCIRP infected group, $n=9$; A549/Le-shCTNNB1 infected group, $n=9$; H460/Le-scrambled infected group, $n=$ 10; H460/Le-shCIRP infected group, $n=10$ ). Twenty-eight days later, animals were sacrificed to harvest the tumor tissues to weight and perform immunohistochemistry assay as well as hematoxylin \& eosin staining. All animals received humane care according to the criteria outlined in the "Guide for the Care and Use of Laboratory Animals" prepared by the National Academy.

\section{Statistical analysis}

The log-rank (Mantel-Cox) test was used to evaluate the statistical significance of correlation between CIRP or CTNNB1 expression and the overall survival of NSCLC patients. Independent sample T test or the nonparametric Mann-Whitney test was used accordingly to study the relationship between CIRP or CTNNB1 expression and other variables. We used Spearman rank test to analyze correlations between variables. The values of qRT-PCR, cell growth rate, cell cycle percentage, aggressive cell numbers, and colony formation were presented as means $\pm S D$, and compared at a given time point by a two-tailed independent sample T-test. Data were considered to be statistically significant when * $P<0.05$ and $* \star P<$ 0.01 .

\section{Other methodologies are detailed in Supplementary information}

\section{Results}

Elevated Expression of CIRP in NSCLC Samples Correlates with Poor Prognosis.

To investigate expression of CIRP in NSCLC specimens, we measured mRNA level of CIRP in eight fresh NSCLC samples and their adjacent non-NSCLC tissues. Our data showed that the mRNA abundance of CIRP was significantly higher in NSCLC tissues than non-NSCLC tissues (Fig. 1A). We confirmed the elevated CIRP expression in NSCLC samples at protein level by immunoblotting (Fig. 1B). Immunohistochemical staining showed an increased expression of CIRP in cancerous tissue. CIRP protein was localized in both the nuclei and plasma of cancer cells (Fig. 1C). These findings revealed an observably upregulation of CIRP in NSCLC. Subsequently, we investigated whether aberrant expression of CIRP predicts prognosis in NSCLC patients. We generated a tissue array containing 86 NSCLC samples and their corresponding noncancerous lung tissues to determine CIRP expression level by immunohistochemical staining. The representative images of NSCLC and noncancerous samples with strong, moderate or weak CIRP expression were shown in Fig. 1D. There were 55.8\% of NSCLC samples showing strong CIRP expression, while the percentages of moderate and weak CIRP expression were $24.4 \%$ and $19.8 \%$, respectively; In contrast, CIRP expression was relatively low in noncancerous lung tissues. Only $9.3 \%$ of noncancerous samples had strong CIRP expression, while the percentages of moderate and weak CIRP expression were $36.0 \%$ and $54.7 \%$, respectively (Fig. $1 \mathrm{E}$ ). Clinicopathological 
analysis showed that overexpression of CIRP in NSCLC was correlated with T stage $(P=0.039)$ and increased incidence of death $(P=0.002)$ as well as the lymph node metastasis $(P=0.023)$

(Supplementary Table S1). Importantly, CIRP high-expression patients had shorter total survival than CIRP low-expression patients $(\mathrm{P}=0.0006)$ (Fig. 1F).

CIRP Promotes the Growth of NSCLC Cells.

CIRP expression pattern was evaluated in lung cancer cell lines at protein level. HaCaT cell line was used as a positive control because it has been validated to express CIRP (9). Relatively high expression level of CIRP protein was detected in A549, H460 and cisplatin resistant H446 (H446-CDDP) cell lines, but not in H1299 and H1650 cell lines (Fig. 2A). Our data showed that the CIRP high-expressed cells (A549 and H460) had increased growth rates than CIRP low-expressed cells (H1299 and H1650) (Fig. 2B).

Correlation analysis showed an inverse correlation between relative CIRP protein expression and doubling time of proliferation in these NSCLC cell lines (Fig. 2C). These results indicated a proliferation-promoting role of CIRP in NSCLC cells. To further verify this finding, we synthesized three siRNAs that specifically targeted the CIRP mRNA. Our data showed that siRNA 214 and siRNA 292 distinctly inhibited the expression of CIRP, while siRNA 558 had a relatively weak effect of silencing CIRP expression in A549 cells (Supplementary Fig. 1A). By combination of siRNA 214 and siRNA 292, the expression of CIRP was robustly knocked down in both A549 and H460 cells (Supplementary Fig. 1B). We found that silencing CIRP inhibited the growth of A549 and H460 cell lines (Fig. 2D). On the other hand, we infected CIRP lowexpressed H1299 and H1650 cells with lentiviral vector expressing CIRP (Le-CIRP) to evaluate its growth promoting effect on NSCLC cells. After infection of H1299 and H1650 cells with Le-CIRP, the cells expressed high level of exogenous CIRP (Supplementary Fig. 1C). Le-CIRP-infected H1299 and H1650 cells exhibited higher proliferation rates, as compared with control vector Le-control-infected cells (Fig. 2E). Consistently, Cell cycle analysis revealed that silencing CIRP expression in A549 and H460 cells caused G1/S arrest (Fig. 2F and Supplementary Fig. 2A). overexpression of CIRP in H1299 and H1650 cells resulted in the accelerated G1/G0 to S transition (Fig. 2G and Supplementary Fig. 2B).

CIRP Promotes the Aggressive Capability of NSCLC Cells.

In order to further study the biological function of CIRP in NSCLC cells, we analyzed the effect of CIRP on the migration and invasion ability of NSCLC cells. Our data showed that silencing CIRP with siRNA214/292 inhibited the migration of A549 and H460 cells compared with the NS control siRNA (Fig. 3A). Le-CIRP-infected H1299 and H1650 cells exhibited higher migration ability compared with Lecontrol-infected cells (Fig. 3B). Consistently, we also found that silencing CIRP inhibited the invasion capability of A549 and H460 cell lines (Fig. 3C), while Le-CIRP-infected H1299 and H1650 cells displayed increased invasion capacity in comparison with Le-control-infected cells (Fig. 3D). These results suggested a metastasis-promoting role of CIRP in NSCLC cells. To further confirm this observation, we constructed a lentiviral vector carrying short hairpin RNA (shRNA) that specifically knocked down CIRP expression (Le-shCIRP) and a scrambled control lentiviral vector (Le-Scrambled) (10). We found that infection of A549 and H460 cells with Le-shCIRP at multiplicity of infection (MOI) of 10 inhibited CIRP 
expression more efficiently, as compared with infection of cells with Le-shCIRP at MOI of 1

(Supplementary Fig. 3A and B). Thus, we used a MOI of 10 at the following experiments. The role of CIRP in metastasis of NSCLC cells was also investigated in animal model. We infected luciferase stably expressed A549 (A549-Luc ${ }^{+}$) cells with Le-shCIRP or Le-Scrambled. At 72 h after infection, A549-Luc ${ }^{+}$ cells $\left(1 \times 10^{5}\right)$ were intravenously injected via the tail vein to establish metastatic model. Our data showed that CIRP downregulation resulted in a significant reduction of pulmonary space-occupying lesions by bioluminescence imaging (Fig. 3E) and quantification of the luciferase activity at different time points (Fig. 3F). Compared with nude mice injected with Le-Scrambled A549-Luc ${ }^{+}$cells, the mice bearing tumors derived from CIRP-depleted A549-Luc ${ }^{+}$cells showed significantly prolonged survival assessed by Kaplan-Meier survival curves $(P=0.00117)$ (Fig. 3G).

CIRP Promotes the Tumorigenesis of NSCLC Cells.

We found that the efficiency of colony formation was decreased when A549 and H460 cells were infected with Le-shCIRP (Fig. 4A), while Le-CIRP-infected H1299 and H1650 cells displayed the increased colony forming capacity in comparison with Le-control-infected counterparts (Fig. 4B). The role of CIRP in tumor formation of NSCLC cells was also investigated in animal models. Our data showed that the tumors derived from Le-shCIRP-infected A549 and H460 cells were much smaller than that derived from Lescrambled infected A549 and H460 cells, respectively (Fig. 4C and Supplementary Fig. 4A). The average weight of tumors was significantly lower in Le-shCIRP-infected group than that in Le-scrambled infected group ( $P=0.0026$ and $P=0.005$ ) (Fig. 4D and Supplementary Fig. 4B). Immunohistochemical staining analysis revealed extensive expression of CIRP in tumors from Le-scrambled-infected group, whereas CIRP expression was barely detectable in the formed tumors from Le-shCIRP-infected group (Fig. 4E and Supplementary Fig. 4C). These data indicated that CIRP regulates tumor formation both in vitro and in vivo.

CIRP Regulates the Wnt/ $\beta$-Catenin Signaling Pathway in NSCLC Cells.

To elucidate the mechanisms by which CIRP promotes proliferation, metastasis and tumor formation of NSCLC cells. We examined global gene expression profiles in A549 cells after transfection with siRNA214/292 against CIRP and control siRNA by cDNA microarray. By analysis of the data, we found that there were 1194 genes up-regulated and 585 genes down-regulated with 2-fold or higher change in their expression when CIRP was knocked down. Kyoto Encyclopedia of Genes and Genomes (KEGG) systematic analysis (23) revealed these genes enriched in the categories of various cell processes, including DNA replication, cell cycle, Wnt signaling pathway, ECM-receptor interaction, Fanconi anemia pathway, TNF signaling pathway, MAPK signaling pathway and so on (Fig. 5A). Considering the relatively high enrichment of Wnt signaling and its important role in the development of cancer, we next focused on investigating the relationship between CIRP and Wnt signaling pathway. According to the sequencing data, 20 candidate genes were down-regulated by silencing CIRP expression (Fig. 5B). Further qRT-PCR analysis indicated that among these $\mathrm{Wnt} / \beta$-Catenin signaling promoting genes, only CTNNB1 was found to be decreased in CIRP-silenced A549 cells, as compared with the control A549 cells; Wnt/ $\beta$-Catenin 
signaling inhibiting genes such as LRP4 and APC2 were also down-regulated; Meanwhile, mRNA abundance of Wnt/ $\beta$-Catenin signaling target genes including COX-2, CCND1, MMP7, CD44, AXIN2, TCF7 and VEGFA was reduced when CIRP expression was depressed in A549 cells (Fig. 5C). These findings suggested that CIRP might mediate the canonical Wnt pathway by regulating the expression of CTNNB1. Immunoblotting analysis further confirmed that the protein level of CTNNB1, C-myc, COX-2, CCND1, MMP7, VEGFA and CD44 was reduced in CIRP-silenced A549 and H460 cells, as compared with NS control cells (Fig. 5D). On the contrary, infection of H1650 and H1299 cells with Le-CIRP resulted in the increased protein level of these genes comparing with Le-control infected H1650 and H1299 cells (Fig. 5E).

Overexpression of CTNNB1 in Cancer Cells Correlates with Poor Prognosis of Patients with NSCLC.

To investigate whether aberrant expression of CTNNB1 predicts prognosis in NSCLC patients, we used the same tissue array to determine CTNNB1 expression level by immunohistochemical staining. Our data showed that strong, moderate or weak CTNNB1 expressions were observed in both NSCLC and noncancerous tissues (Supplementary Fig. 5A). There were 45.3\% of NSCLC samples showing strong CTNNB1 expression, while the percentages of moderate and weak CIRP expression were $36.1 \%$ and $18.6 \%$, respectively (Supplementary Fig. 5B). In contrast, CTNNB1 expression was relatively low in noncancerous lung tissues. About 22.1\% of noncancerous samples had strong CTNNB1 expression, while the percentages of moderate and weak CTNNB1 expression were $62.8 \%$ and $15.1 \%$, respectively (Supplementary Fig. 5C). Clinicopathological analysis showed that elevated expression of CTNNB1 in NSCLC was correlated with Tumor stage $(P=0.026)$, increased incidence of death $(P=0.024)$, and lymph node metastasis $(P=0.007)$ (Supplementary Table S1). Similar with CIRP, CTNNB1 highly-expressed patients had shorter total survival than lowly-expressed patients $(P=0.0063)$ (Supplementary Fig. 5D).

Knockdown of CTNNB1 Suppressed Tumorigenesis of NSCLC Cells.

Next, we investigated the role of CTNNB1 in tumor formation of NSCLC cells in animal model. We constructed a lentiviral vector carrying shRNA that specifically knocked down CTNNB1 expression (LeshCTNNB1) (24). Le-shCTNNB1 at MOI of 10 infected A549 cells formed small tumors in $78 \%$ of nude mice. In contrast, Le-scrambled infected cells formed tumors in $100 \%$ of nude mice, and the volumes of tumors were much larger (Supplementary Fig. 6A). The average weight of tumors was significantly lower in Le-shCTNNB1-infected group than that in Le-scrambled infected group $(P=0.0008)$ (Supplementary Fig. 6B). By immunohistochemical staining analysis, we observed an extensive expression of CTNNB1 in tumors derived from Le-scrambled-infected group, while CTNNB1 expression was quite weak in the tumors derived from Le-shCTNNB1-infected group (Supplementary Fig. 6C). These data showed that CTNNB1 silence suppressed NSCLC formation in vivo.

Association of CIRP and CTNNB1 in NSCLC Tissues.

To further clarify the regulatory relationship between CIRP and CTNNB1, we next detected mRNA level of CTNNB1 in the eight fresh NSCLC samples and paired noncancerous tissues. Similar with CIRP 
expression, the mRNA abundance of CTNNB1 was significantly higher in NSCLC tissues than that in noncancerous tissues (Fig. 6A). By analyzing the mRNA abundance of CIRP and CTNNB1, we found that CIRP and CTNNB1 were positively correlated in these cancer samples (Fig. 6B). In our research, we were surprised to find that the expressions of CIRP and CTNNB1 were very similar according to Spearman's correlation. By analyzing the immunohistochemical staining of CIRP and CTNNB1 in tissue array, we found CTNNB1 expression was positively associated with CIRP expression $(P<0.001)$ (Fig. 6C). Although this correlation is statistically significant, it is worth pondering whether CIRP directly regulates CTNNB1 in NSCLC cells and what mechanism underlies this regulation.

CIRP Increases CTNNB1 mRNA Stability and Protein Translation in NSCLC Cells.

To elucidate the mechanism by which CIRP regulates CTNNB1 expression in NSCLC cells, we implemented the RNA-IP experiments. Our data showed that both pairs of CTNNB1 mRNA detection primers could successfully amplify the target fragments in the anti-CIRP IP group, as well as in the input positive control group (Supplementary Fig. 7A). In addition, qRT-PCR analysis revealed that anti-CIRP IP group successfully enriched CTNNB1 mRNA by about 76 folds, as compared with the input control group (Fig. 7A). Furthermore, biotin pull-down assay was performed to further confirm the interaction of CIRP and CTNNB1 mRNA. Our data showed a large amount of CIRP protein was associated with the 5'-UTR (Untranslated region) and 3'-UTR of CTNNB1 mRNA, nevertheless, the negative control transcripts failed to pull down CIRP (Fig. 7B). Next, RNA decay analyses were performed in the presence of actinomycin D (a transcriptional inhibitor, $15 \mu \mathrm{g} / \mathrm{mL}$ ). Our results showed that knockdown of CIRP by siRNA led to the accelerated decay of CTNNB1 mRNA (Fig. 7C).

In order to further explore the combination of CIRP with the 5'-UTR and 3'-UTR of CTNNB1 mRNA, we constructed a pC3.1/Control reporter vector by replacing the original neomycin gene with renilla luciferase $(R L)$ coding DNA fragment and inserting the firefly luciferase $(F L)$ coding DNA fragment into the multiple cloning site (MCS) of pcDNA3.1. Thereafter, the 5'-UTR and 3'-UTR coding DNA were respectively cloned into the flank of the FL coding region to form pC3.1/5'-UTR report and pC3.1/3'-UTR reporter vectors (Fig. 7D). Moreover, a pC3.1/CDS reporter vector was also created by fusion expression of FL-CTNNB1 protein (Supplementary Fig. 7B). After transfection of each reporter vectors into A549 cells for $72 \mathrm{~h}$, RNAIP experiments were performed. Our results showed that anti-CIRP IP groups successfully enriched FL mRNA in pC3.1/5'-UTR reporter- and pC3.1/3'-UTR reporter-transfected cells, but not in pC3.1/Control reporter- and pC3.1/CDS reporter-transfected cells (Fig. 7E and Supplementary Fig. 7C). These data suggested that CIRP could directly bind to the 5'-UTR and 3'-UTR of CTNNB1 mRNA in NSCLC cells. Then, RNA decay analyses were performed in each reporter vector and CIRP siRNA or NS siRNA co-transfected A549 cells. qRT-PCR analysis revealed that silencing of CIRP expression resulted in the accelerated degradation of CTNNB1 3'-UTR-foused FL mRNA, while the decay of FL control mRNA and CTNNB1 5'UTR or CTNNB1 CDS fused FL mRNA was no affected by CIRP knockdown (Fig. 7F and Supplementary Fig. 7D). In addition, luciferase activity assay revealed that CIRP silencing resulted in the decreased of relative luciferase activity in pC3.1/5'-UTR reporter- and pC3.1/5'-UTR reporter-transfected A549 cells, whereas no significant inhibitory effect was observed in A549 cells transfected with pC3.1/Control 
reporter or pC3.1/CDS reporter vectors when CIRP expression was silenced (Fig. 7G and Supplementary Fig. 7E). It's worth noting that without changing the CIRP expression, the relative luciferase activity in A549 cells transfected with $\mathrm{pC} 3.1 / 5^{\prime}$-UTR reporter or $\mathrm{pC} 3.1 / 3^{\prime}$-UTR reporter vectors was higher than that in pC3.1/CDS reporter-transfected cells (Fig. 7G). These results suggested that CIRP bound to the 3'-UTR of CTNNB1 mRNA to increase its stability and facilitate its translation in NSCLC cells.

CIRP Promotes IRES-dependent Translation of CTNNB1 in NSCLC Cells.

Our data also indicated that CIRP binds to the 5'-UTR of CTNNB1 mRNA to promote the protein translation. Nevertheless, the exact molecular mechanism has not been illustrated. It has been reported that the 5'-UTR of CTNNB1 mRNA contained an internal ribosome entry segment (IRES) that regulated its translation (25). Given that CIRP is a known RNA-binding protein, and its main role in the cytoplasm is to bind specific mRNAs (IRES-contained mRNAs) to facilitate their translation upon stresses (7), we wonder whether the cytoplasmic CIRP of NSCLC cells would also promote IRES-dependent translation of CTNNB1. To verify our conjecture, we did biotin pull-down assay in A549 cells. We found that the $5^{\prime}$-UTR of CTNNB1 mRNA was able to pull down the proteins that were closely related to IRES-dependent translation process, such as LARP3, PTBP1, YB-1, elF4E and elF2a (Fig. 7H). Further Immuno-purification assay demonstrated that all the above indicated proteins were co-purified with CIRP from cytoplasmic extracts of A549 cells (Fig. 7l, RNaseA-). However, the copurification was abolished or severely reduced in the presence of RNaseA (Fig. 7I, RNaseA +). Additionally, knockdown of the IRES trans-acting factors (ITAFs: LARP3, PTBP1 and YB-1) separately with their siRNAs resulted in reductions of CTNNB1 protein in A549 cells, while to our surprise, the expression of CIRP was also reduced (Fig. 7J). These findings suggested that CIRP directly interacted with CTNNB1 via binding to its 5'-UTR to promote the IRESdependent protein translation in NSCLC cells.

\section{Discussion}

CIRP has been linked to tumor growth and metastasis as an oncogene in several types of human cancer $(9,18,26-28)$. However, the exact function(s) of CIRP on the regulation of critical cellular activities and progression of NSCLC remain unclear. We demonstrated here that CIRP was overexpressed in $55.8 \%$ NSCLC tissues and was correlated with more frequent lymph node metastasis and shorter survival, indicating that CIRP expression level is of prognostic relevance. Our data also demonstrated that there is a positive correlation between CIRP and CTNNB1 expression in NSCLC cells, and CIRP regulates CTNNB1 post-transcriptionally by binding to the 5'-UTR and 3'-UTR of CTBBN1 mRNA. In addition to enhancing CTNNB1 mRNA stability, CIRP could also facilitate IRES-dependent translation to promote CTNNB1 accumulation in NSCLC cells.

Interestingly, we observed that there was a correlation between CIRP expression level and cell growth rate. Moreover, knockdown of CIRP in CIRP high-expressed NSCLC cells suppressed cell growth, whereas overexpression of CIRP in CIRP low-expressed NSCLC cells accelerated their proliferation. More importantly, knockdown of CIRP in NSCLC cells significantly suppressed their tumorigenicity both in vitro 
and in vivo. Consistent with our findings in NSCLC, CIRP has also been shown to contribute to cell growth in other tumors. Lu et al. demonstrated that CIRP is required for HIF-1a-induced proliferation of human bladder cancer cells (18). Zhou et al. showed that silencing CIRP with siRNA inhibited the proliferation of the 786-0 cells and enhanced its chemosensitivity (28). This function of CIRP has also been reported in UVB-induced skin carcinogenesis (10). Our data that knockdown of CIRP in CIRP high-expressed NSCLC cells induced cell cycle blockade at the G1/S checkpoint, while overexpressing CIRP in CIRP lowexpressed NSCLC cells accelerated G1/G0 to $S$ transition further support the pro-growth effect of CIRP in NSCLC. CCND1 gene codes a well-known G1/S-specific cyclin (Cyclin D1) that promotes the transition of cell cycle from $\mathrm{G} 1$ to $S$ phase (29). In addition, the proliferation-promoting function of CIRP was further verified by our finding that the expression of CCND1 was positively correlated with CIRP expression.

Beside its growth promoting effects, CIRP has been shown to promote tumor metastasis in several types of cancers. Wang et al. demonstrated that CIRP expression was significantly upregulated in pituitary adenoma and contributed to tumor invasion (30). Lee et al. reported that CRIP contributed to TGF- $\beta 1$ induced EMT in human lung carcinoma A549 and hepatocellular carcinoma Huh7 cells (31). Here our clinicopathological analysis showed that CIRP overexpression was correlated with increased incidence of lymph node metastasis in NSCLC. Our in vitro experimental data demonstrated that knockdown of CIRP in CIRP high-expressed NSCLC cells suppressed cell migration and invasion, and accordantly overexpressing CIRP in CIRP low-expressed NSCLC cells enhanced cell migration and invasion. Moreover, knockdown of CIRP in A549-Luc ${ }^{+}$cells reduced the numbers of pulmonary space-occupying lesions when these cells were administered to mice via tail vein injection. Although all these studies support an aggression-promoting function of CIRP in tumors, there is one study in which CIRP has been shown to suppress cell migration in BEV-treated glioma cells (32). This discrepancy may be due to cell type differences, however future studies are needed to explore the distinct functions of CIRP in different diseases.

Wnt/ $\beta$-catenin pathway plays a central role in the development of many human cancers through regulating cell proliferation, migration, and invasion (33-35). As the core factor in the canonical Wnt/ $\beta$ catenin pathway, CTNNB1 can combine E-cadherin to form a cadherin/catenin complex and maintain cell-cell adhesion (36). When the Wnt signaling pathway is activated, CTNNB1 accumulation will occur in the nucleoplasm of tumor cells, leading to the loss of epithelial structural integrity and increased tumor invasion and metastasis $(37,38)$. In addition, CTNNB1 can act as a signal transduction molecule and its activation significantly stimulates the production of VEGF, up-regulates the expression of MMPs to enhance the degradation of extracellular matrix (ECM), and thus promotes tumor cell growth, invasion and metastasis (39). CTNNB1 has been demonstrated to be oncogenic in several tumor types and its expression is regulated by multiple Wnt molecules (40-42). Previous studies have shown that Wnt/ $\beta$ catenin signaling promoters such as Wnt1, Wnt2 and Wnt3a are associated with tumor proliferation and angiogenesis in NSCLC (43-46). Chuan et al. have demonstrated that the transcription of CTNNB1 is directly regulated by Wnt-1 in oral cancer (47). A very recent study has also reported that nuclear abnormal expression of CTNNB1 is associated with mutations of CTNNB1 gene in lung cancer (48). Wu 
et al. have shown that the polyubiquitination and degradation of TRAF6 induces the accumulation of CTNNB1, which contributes to aberrant activation of Wnt/ $\beta$-catenin signaling and thereby promotes the epithelial-mesenchymal transition and metastasis (EMT) of colorectal cancer (CRC)(49). Here our data show that increased CTNNB1 expression in tumor tissues is correlated with poor prognosis in NSCLC patients. Moreover, the expression of CTNNB1 and the downstream target genes of Wnt/ $\beta$-catenin signaling are positively correlated with CIRP expression in NSCLC cells.

CIRP belongs to the family of RNA binding proteins and can specifically bind the 3'-UTRs of mRNAs to stabilize target mRNAs and prolong their translation lifetimes. In human bladder cancer cells, CIRP has been shown to bind the 3'-UTR of HIF-1 a mRNA, thereby increasing its stability and extending its translation (18). Yang et al. have reported that hypothermia induces an airway inflammatory response through CIRP-mediated increases in pro-inflammatory cytokine mRNAs stability and protein translation (50). Consistent with these findings, our data here demonstrate that the binding of CIRP protein with 3'UTR of CTNNB1 mRNA enhances mRNA stability and increases protein translation. It is worth pointing out that CIRP protein can also bind the 5'-UTR of CTNNB1 mRNA and this binding enhances protein translation, but has no significant effect on mRNA stability. To further determine how CIRP - CTNNB1 mRNA 5'-UTR binding promotes CTNNB1 translation, we investigated the interaction of CIRP with other translation-associated factors. Our data show that CIRP can bind to translation initiation complex-related proteins (elF4E and elF2a) and IRES trans-acting factors (LARP3, PTBP1 and YB-1). Since CTNNB1 mRNA contains an IRES sequence in its 5'-UTR (25), we hypothesized that CIRP might bind to 5'-UTR of CTNNB1 to promote an IRES-dependent translation in NSCLC cells. We conducted immuno-purification assays and verified our speculation. Recently, Liu et al have demonstrated that m6A mRNA methylation significantly contributes to the expression and stability of the CTNNB1 in hepatoblastoma(51). Whether and how CIRP interacts with m6A methylation to regulate CTNNB1 mRNA stability is an important question we will answer in future studies. Interestingly, our data show that CIRP is downregulated in A549 cells when we knocked down IRES trans-acting factors LARP3, PTBP1 or YB-1 with siRNAs. One possible explanation is that CIRP mRNA also contains an IRES sequence, and its translation is also partly subject to IRES-dependent translation process(52).

\section{Conclusions}

In summary, as shown in mechanistic diagram (Fig. 8), our study demonstrates that CIRP acts as a tumor promoting gene to promote cell proliferation and aggression in NSCLC both in vitro and in vivo.

Mechanistically, cytoplasmic CIRP increases CTNNB1 expression by binding to the 5'-UTR and 3'-UTR of CTNNB1 mRNA to increase its mRNA stability and facilitate its IRES-dependent translation in NSCLC cells. As a result, the elevated CTNNB1 expression continuously activates the Wnt/ $\beta$-catenin signaling pathway, thereby promoting downstream gene expression and tumor progression. Our data suggest that CIRP may be a useful prognostic marker and a potential therapeutic target for NSCLC.

\section{Abbreviations}


CIRP

Cold-inducible RNA binding protein; NSCLC:Non-small cell lung cancer; RRM:RNA recognition motif; TNFa:Tumor necrosis factor-alpha; ERK1/2:Extracellular signal-regulated kinase 1/2; HIF-1:Hypoxia inducible transcriptional factor-1; VEGF:Vascular endothelial growth factor; RL:Renilla luciferase; FL:Firefly luciferase; IRES:Internal ribosome entry segment; ITAFs:IRES trans-acting factors; UTR:Untranslated region. ECM:Extracellular matrix; EMT:epithelial-mesenchymal transition and metastasis; CRC:colorectal cancer.

\section{Declarations}

\section{Ethics approval and consent to participate}

The study protocol has been approved by the Ethics committee of the First Affiliated Hospital of Third Military Medical University, PLA (2015). All patients signed an informed consent form and volunteered to participate in this study.

\section{Consent for publication}

Not Applicable

\section{Availability of data and materials}

All data generated or analyzed during this study are included in this published article [and its supplementary information files].

\section{Competing interests}

The authors of this article declared they have no potential conflict of interests.

\section{Funding}

This work was supported by funds from the National Natural Sciences Foundation of China (No. 81702921 to Yi Liao), China Postdoctoral Science Foundation Grant (No. 2019M663106 to Yi Liao) and Shenzhen Science and Technology Commission (JCYJ20170817172241688 and JCYJ20170817172150505 to Xiufeng Ye, JCYJ20170413104646428 to Wenlan Liu).

\section{Authors' Contributions}

YL and JF contributed equally to this study, performing the experiments and preparing the first draft of the manuscript. WS and CW assisted in cell culture and fed the animal models. JL and TJ helped in data analysis. YL assisted in data analysis. YQ, WL and HW instructed the study and finalized the manuscript. All authors read and approved the final manuscript.

\section{Acknowledgements}


Thanks for the experimental platform provided by the Institute of Burn Research, South West Hospital, AMU (TMMU).

\section{References}

1. Siegel RL, Miller KD, Jemal A. Cancer statistics, 2018. Ca A Cancer Journal for Clinicians 2018;68:7

2. Luca T, Sabrina R, Giovanna F, Armando S. Non-small cell lung cancer treatment (r)evolution: ten years of advances and more to come. Ecancermedicalscience 2017;11

3. Zhou, Qing. JCES01.04 Liquid Biopsy in Monitoring Dynamic Changes of Driver Genes in Advanced NSCLC. Journal of Thoracic Oncology 2017;12:S232-S3

4. Shi X, Chen Y, Feng F, Chen J, Wen S, Li J. P1.07-005 A Systematic and Genome-Wide Correlation Analysis of PD-L1 Expression and Common NSCLC Driver Genes. Journal of Thoracic Oncology 2017;12:S1996-S7

5. Wang H, Wang Z, Zhang G, Zhang M, Ma Z. Driver genes as predictive indicators of brain metastasis in patients with advanced NSCLC: EGFR, ALK, and RET gene mutations. Cancer Medicine 2019;9

6. Hui X, Xi L, Chunshui Z. Screening of lung cancer tumor-associated suppressor genes using shRNA library targeting human transcriptome. Practical Oncology Journal 2019

7. Yi, Liao, Lingying, Tong, Liling, Tang, et al. The role of cold-inducible RNA binding protein in cell stress response. International Journal of Cancer 2017

8. Sheikh MS, Carrier F, Papathanasiou MA, Hollander MC, Zhan Q, Yu K, et al. Identification of Several Human Homologs of Hamster DNA Damage-inducible Transcripts: CLONING AND CHARACTERIZATION OF A NOVEL UV-INDUCIBLE CDNA THAT CODES FOR A PUTATIVE RNABINDING PROTEIN. Journal of Biological Chemistry 1997;272:26720-6

9. Sun W, Yi L, Qian Y, Wu S, Tang L, Tong L. The Mechanism of CIRP in Regulation of STAT3 Phosphorylation and Bag-1/S Expression Upon UVB Radiation. Photochemistry \& Photobiology 2018

10. Liao Y, Feng J, Zhang Y, Tang L, Wu S. The mechanism of CIRP in inhibition of keratinocytes growth arrest and apoptosis following low dose UVB radiation. Molecular Carcinogenesis 2016

11. Wang G, Zhang JN, Guo JK, Cai Y, Sun HS, Dong K, et al. Neuroprotective effects of cold-inducible RNA-binding protein during mild hypothermia on traumatic brain injury. Neural Regen Res 2016;11:771-8

12. Cen C, Mcginn J, Aziz M, Yang WL, Cagliani J, Nicastro JM, et al. Deficiency in cold-inducible RNAbinding protein attenuates acute respiratory distress syndrome induced by intestinal ischemiareperfusion. Surgery 2017:S003960601730394X

13. Artero-Castro A, Callejas FB, Castellvi J, Kondoh H, Carnero A, Fernández-Marcos PJ, et al. Coldinducible RNA-binding protein bypasses replicative senescence in primary cells through extracellular signal-regulated kinase 1 and 2 activation. Mol Cell Biol 2009;29:1855-68

14. Li S, Zhang Z, Xue J, Liu A, Zhang H. Cold-inducible RNA binding protein inhibits H2O2-induced apoptosis in rat cortical neurons. Brain Research 2012;1441:47-52 
15. Lujan, Daniel, A., Ochoa, Joey, L., et al. Cold-inducible RNA binding protein in cancer and inflammation. Wiley Interdisciplinary Reviews Rna 2018

16. Noike T, Miwa S, Soeda J, Kobayashi A, Miyagawa SI. Increased expression of thioredoxin-1, vascular endothelial growth factor, and redox factor- 1 is associated with poor prognosis in patients with liver metastasis from colorectal cancer. Human Pathology 2008;39:0-208

17. Rohwer, N., Lobitz, S., Daskalow, K., et al. HIF-1a determines the metastatic potential of gastric cancer cells. British Journal of Cancer 2009

18. Mengxin, Lu, Qiangqiang, Ge, Gang, Wang, et al. CIRBP is a novel oncogene in human bladder cancer inducing expression of HIF-1a. Cell death \& disease 2018

19. Zhang Q, Wang Y-Z, Zhang W, Chen X, Wang J, Chen J, et al. Involvement of Cold Inducible RNABinding Protein in Severe Hypoxia-Induced Growth Arrest of Neural Stem Cells In Vitro. Molecular Neurobiology 2016;54:2143-53

20. Liu J, Xue J, Zhang H, Li S, Liu Y, Xu D, et al. Cloning, expression, and purification of cold inducible RNA-binding protein and its neuroprotective mechanism of action. Brain Research 2015;1597:189-95

21. Cold-inducible RNA-binding protein, CIRP, inhibits DNA damage-induced apoptosis by regulating p53. Biochemical \& Biophysical Research Communications 2015;464:916-21

22. Xinzhou Zhu, Christoph Bührer, Sven Wellmann. Cold-inducible proteins CIRP and RBM3, a unique couple with activities far beyond the cold. Cellular \& Molecular Life Sciences 2016

23. Kanehisa M, Goto S, Sato Y, Furumichi M, Tanabe M. KEGG for integration and interpretation of largescale molecular data sets. Nucleic Acids Res 2012;40:D109-14

24. Li GQ, Fang YX, Liu Y, Meng FR, Wu X, Zhang CW, et al. MALAT1-Driven Inhibition of Wnt Signal Impedes Proliferation and Inflammation in Fibroblast-Like Synoviocytes Through CTNNB1 Promoter Methylation in Rheumatoid Arthritis. Hum Gene Ther 2019;30:1008-22

25. Fu Q, Chen Z, Gong X, Cai Y, Chen Y, Ma X, et al. $\beta$-Catenin expression is regulated by an IRESdependent mechanism and stimulated by paclitaxel in human ovarian cancer cells. Biochemical \& Biophysical Research Communications 2015;461:21-7

26. Lee HN, Ahn SM, Jang HH. Cold-inducible RNA-binding protein promotes epithelial-mesenchymal transition by activating ERK and p38 pathways. Biochemical \& Biophysical Research Communications 2016:S0006291X16311433

27. Sakurai T, Kashida H, Watanabe T, Hagiwara S, Mizushima T, lijima H, et al. Stress response protein cirp links inflammation and tumorigenesis in colitis-associated cancer. Cancer Res 2014;74:6119-28

28. Zhou KW, Jiang K, Zhu W, Weng G. Expression of cold-inducible RNA-binding protein (CIRP) in renal cell carcinoma and the effect of CIRP downregulation cell proliferation and chemosensitivity to gemcitabine. Oncol Lett 2018;15:7611-6

29. Ramos-García P, Gil-Montoya JA, Scully C, Ayén A, González-Ruiz L, Navarro-Triviño FJ, et al. An update on the implications of cyclin D1 in oral carcinogenesis. Oral Dis 2017;23:897-912 
30. Wang M, Zhang H, Heng X, Pang Q, Sun A. Expression of cold-inducible RNA-binding protein (CIRP) in pituitary adenoma and its relationships with tumor recurrence. Med Sci Monit 2015;21:1256-60

31. Lee HN, Ahn SM, Jang HH. Cold-inducible RNA-binding protein promotes epithelial-mesenchymal transition by activating ERK and p38 pathways. Biochem Biophys Res Commun 2016;477:1038-44

32. Liu YX, Zhou JN, Liu KH, Fu XP, Zhang ZW, Zhang QH, et al. CIRP regulates BEV-induced cell migration in gliomas. Cancer Manag Res 2019;11:2015-25

33. Wang W, Smits R, Hao H, He C. Wnt/ $\beta$-Catenin Signaling in Liver Cancers. Cancers 2019;11

34. Krishnamurthy N, Kurzrock R. Targeting the Wnt/beta-catenin pathway in cancer: Update on effectors and inhibitors. Cancer Treat Rev 2018;62:50-60

35. Stewart DJ. Wnt signaling pathway in non-small cell lung cancer. J Natl Cancer Inst 2014;106:5

36. van Roy F, Berx G. The cell-cell adhesion molecule E-cadherin. Cellular and molecular life sciences : CMLS 2008;65:3756-88

37. Kassouf T, Larive RM, Morel A, Urbach S, Bettache N, Marcial Medina MC, et al. The Syk Kinase Promotes Mammary Epithelial Integrity and Inhibits Breast Cancer Invasion by Stabilizing the ECadherin/Catenin Complex. Cancers 2019;11

38. Bremnes RM, Veve R, Hirsch FR, Franklin WA. The E-cadherin cell-cell adhesion complex and lung cancer invasion, metastasis, and prognosis. Lung Cancer 2002;36:115-24

39. Belaya ZE, Rozhinskaya LY, Melnichenko GA, Solodovnikov AG, Dragunova NV, Iljin AV, et al. Serum extracellular secreted antagonists of the canonical Wnt/beta-catenin signaling pathway in patients with Cushing's syndrome. Osteoporosis international : a journal established as result of cooperation between the European Foundation for Osteoporosis and the National Osteoporosis Foundation of the USA 2013;24:2191-9

40. van Schie EH, van Amerongen R. Aberrant WNT/CTNNB1 Signaling as a Therapeutic Target in Human Breast Cancer: Weighing the Evidence. Frontiers in cell and developmental biology 2020;8:25

41. Nguyen VHL, Hough R, Bernaudo S, Peng C. Wnt/beta-catenin signalling in ovarian cancer: Insights into its hyperactivation and function in tumorigenesis. Journal of ovarian research 2019;12:122

42. Cui C, Zhou X, Zhang W, Qu Y, Ke X. Is beta-Catenin a Druggable Target for Cancer Therapy? Trends Biochem Sci 2018;43:623-34

43. Yong C, Min L, Ren C, Xu X, Yang J, Sun X, et al. miRNA-148a serves as a prognostic factor and suppresses migration and invasion through Wnt 1 in non-small cell lung cancer. Plos One 2017;12:e0171751-

44. Huang C, Liu D, Ishikawa S, Nakano J, Kadota K, Yokomise H. Wnt1 overexpression is associated with tumor proliferation and angiogenesis in non-small cell lung cancer. Cancer Research 2008;68

45. Huang C, Ma R, Xu Y, Li N, Qi D. Wnt2 promotes non-small cell lung cancer progression by activating WNT/ $\beta$-catenin pathway. Am J Cancer Res 2015;5:1032-46

46. Chunyan L, Linjun S, Song G. The regulation of Wnt3a on the cell growth in non-small-cell lung cancer cell lines. Journal of Modern Oncology 2014 
47. Ma C, Shi L, Huang Y, Shen L, Peng H, Zhu X, et al. Nanoparticle delivery of Wnt-1 siRNA enhances photodynamic therapy by inhibiting epithelial-mesenchymal transition for oral cancer. Biomaterials science 2017;5:494-501

48. Thomas de Montpreville V, Lacroix L, Rouleau E, Mamodaly M, Leclerc J, Tutuianu L, et al. Non-small cell lung carcinomas with CTNNB1 (beta-catenin) mutations: A clinicopathological study of 26 cases. Annals of diagnostic pathology 2020;46:151522

49. Wu H, Lu XX, Wang JR, Yang TY, Li XM, He XS, et al. TRAF6 inhibits colorectal cancer metastasis through regulating selective autophagic CTNNB1/beta-catenin degradation and is targeted for GSK3B/GSK3beta-mediated phosphorylation and degradation. Autophagy 2019;15:1506-22

50. Juan Y, Haiqiao W, Xie W, Huaping H, Zhong H, Xiangdong Z, et al. Cold-inducible RNA-binding protein mediates airway inflammation and mucus hypersecretion through a post-transcriptional regulatory mechanism under cold stress. International Journal of Biochemistry \& Cell Biology 2016;78:335-48

51. Liu L, Wang J, Sun G, Wu Q, Ma J, Zhang X, et al. m(6)A mRNA methylation regulates CTNNB1 to promote the proliferation of hepatoblastoma. Mol Cancer 2019;18:188

52. Al-Fageeh MB, Smales CM. Cold-inducible RNA binding protein (CIRP) expression is modulated by alternative mRNAs. RNA 2009;15:1164-76

\section{Figures}


A.

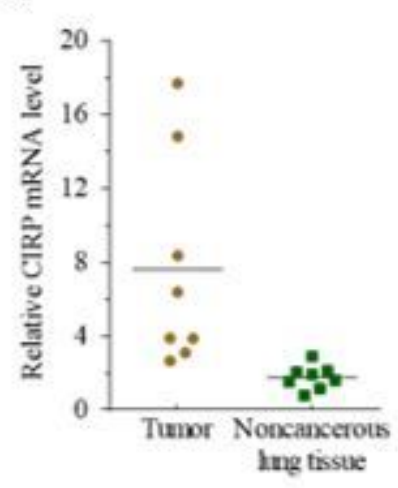

D.

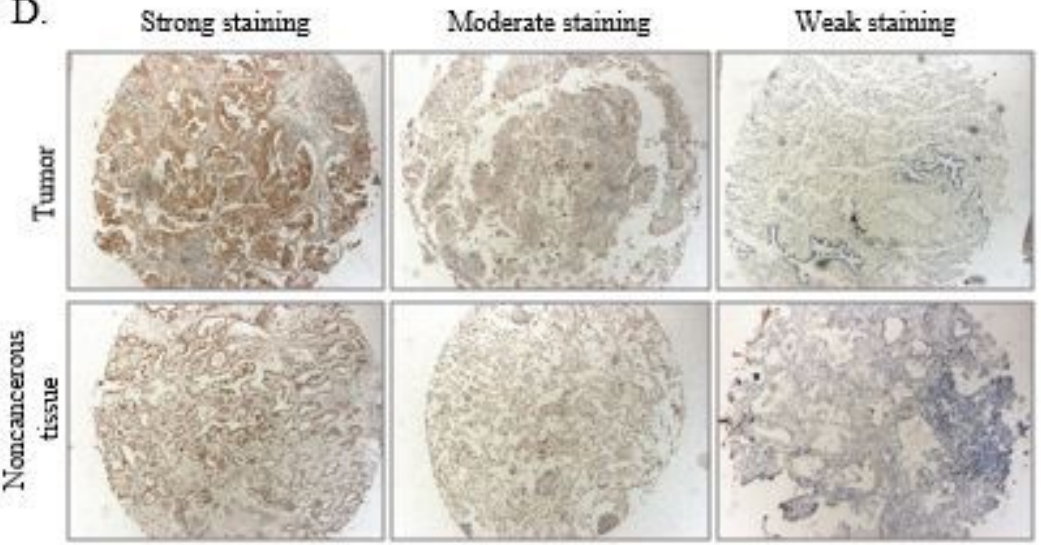

B.

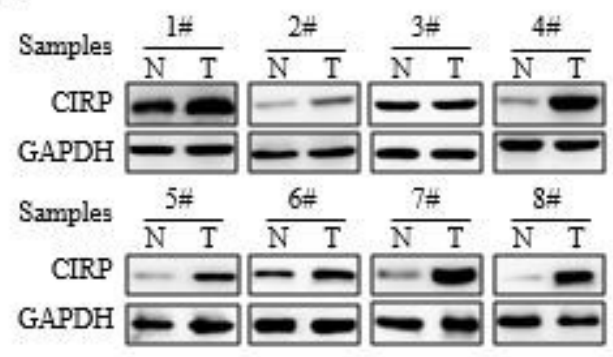

C.

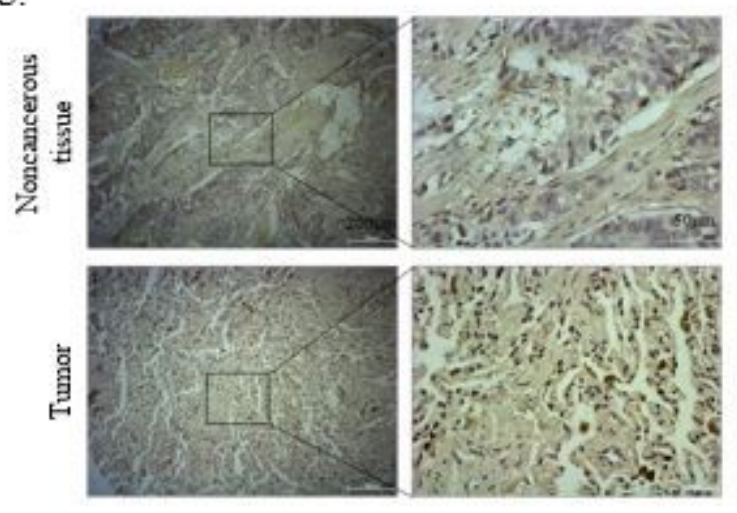

E.

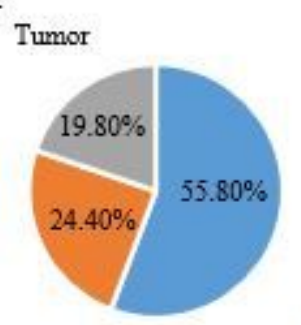

" Strong staining " Weak staining

F.

F. Kaplan-Meier Cum Survival Plot for Lung Cancer

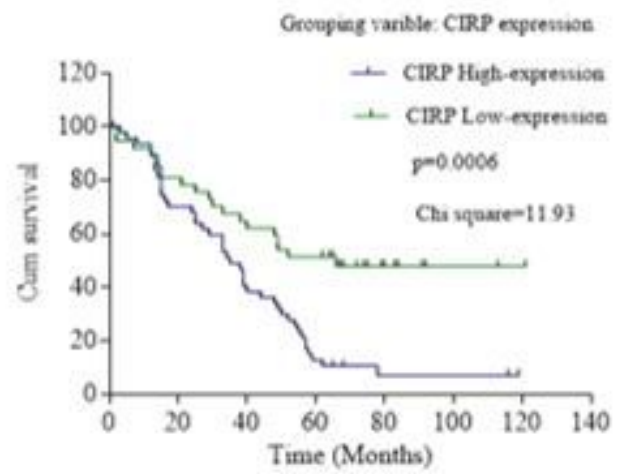

\section{Figure 1}

Elevated Expression of CIRP in NSCLC Samples Correlates with Poor Prognosis. (A) mRNA levels of CIRP were quantified in NSCLC samples and their corresponding noncancerous lung tissues as well as normal lung tissues by qRT-PCR $(n=8)$. CIRP expression was normalized by GAPDH expression and each noncancerous lung tissue was used as a control. Data were shown as mean $\pm S D(\star \star, P<0.01)$. (B) Protein level of CIRP was determined by immunoblotting. GAPDH expression served as endogenous reference. (C) Immunohistochemical analysis on CIRP expression was performed in NSCLC samples and their corresponding noncancerous lung tissues. Representative microphotographs of CIRP expression were displayed. (D) The representative microphotographs showed strong, moderate and weak staining of CIRP in NSCLC tissues and their corresponding noncancerous lung tissues by IHC in tissue array (magnification $\times 40)$. (E) The percentages of strong, moderate and weak CIRP expression in NSCLC 
samples and corresponding noncancerous lung tissues were shown in pie charts. (F) Kaplan-Meier analysis of overall survival of NSCLC patients according to the expression level of CIRP protein in NSCLC tissues (CIRP High-expression, $n=48$ and CIRP Low-expression, $n=38$ ).

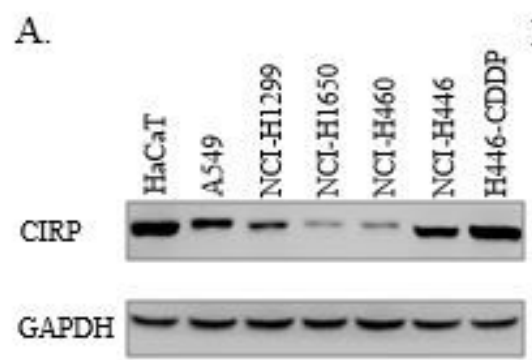

B.

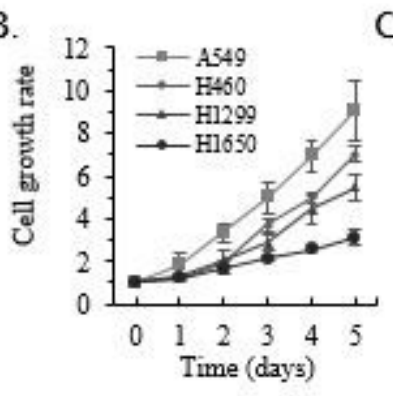

C.

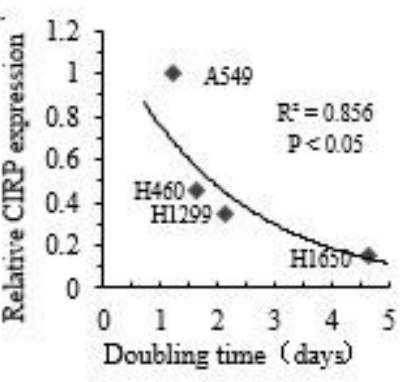

D.

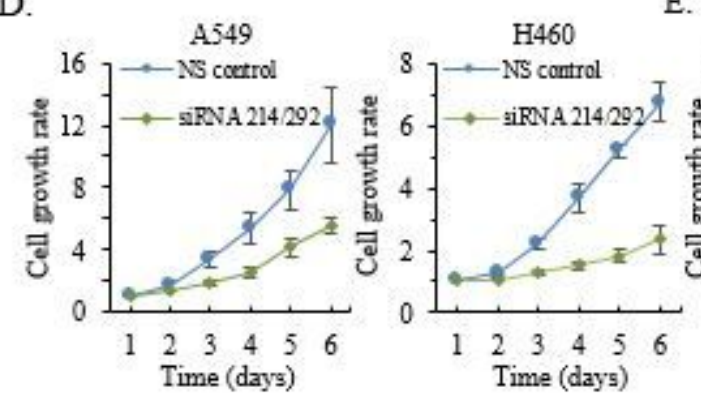

E.
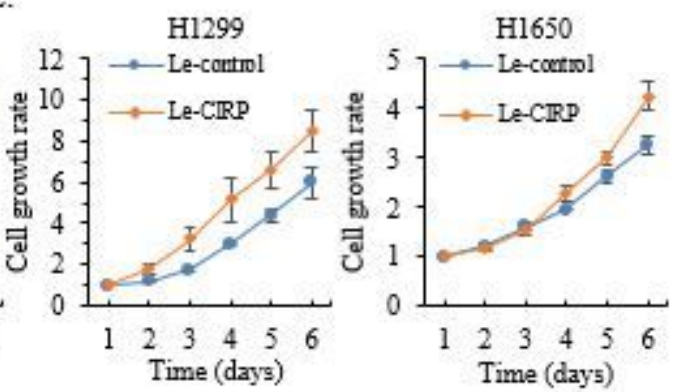

F.
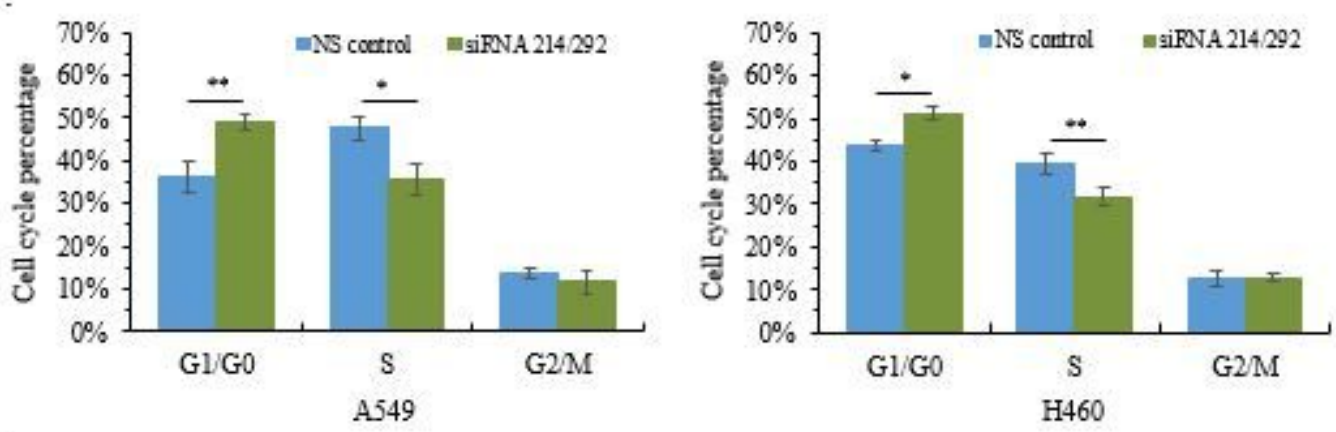

G.

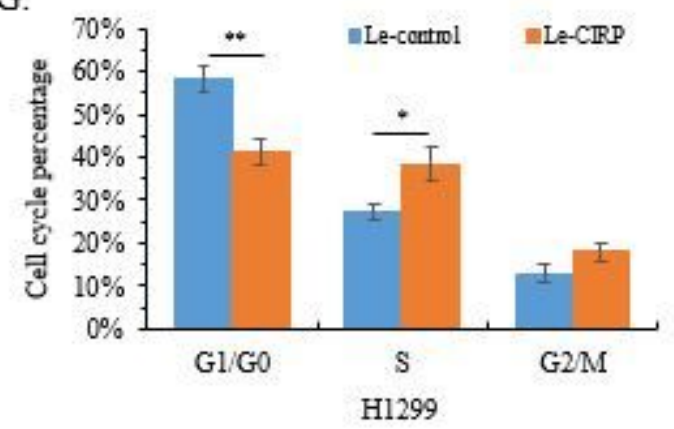

\section{Figure 2}

CIRP Regulates the Growth of NSCLC Cells. (A) Protein level of CIRP in lung cancer cell lines was determined by immunoblotting. HaCaT cell line served as a positive control. (B) Growth rates of NSCLC cell lines with various levels of CIRP expression as determined by cell proliferation assay. Data were means \pm SD from three independent experiments. (C) A significant inverse correlation is shown between 
CIRP protein levels and cell doubling time for these NSCLC cell lines. (D) Growth rates of A549 and H460 cells in which CIRP expression was silenced by siRNA were determined. Data were means \pm SD from three independent experiments. (E) Growth rates of CIRP stably overexpressed H1299 and H1650 cells were determined. Data were means \pm SD from three independent experiments. $(F)$ Cell cycle analysis was performed in $\mathrm{A} 549$ and $\mathrm{H} 460$ cells 72 hours after transfection with indicated siRNAs. The average percentages at $\mathrm{G} 1 / \mathrm{G} 0, \mathrm{~S}$ and $\mathrm{G} 2 / \mathrm{M}$ phases were shown as mean $\pm \mathrm{SD}$ from three independent experiments $(*, P<0.05$ and $* *, P<0.01)$. (G) Cell cycle analysis was performed in CIRP stably overexpressed $\mathrm{H} 1299$ and $\mathrm{H} 1650$ cells. The average percentages at G1/G0, S and G2/M phases were shown as mean \pm SD from three independent experiments ( $*, P<0.05$ and $* *, P<0.01$ ).
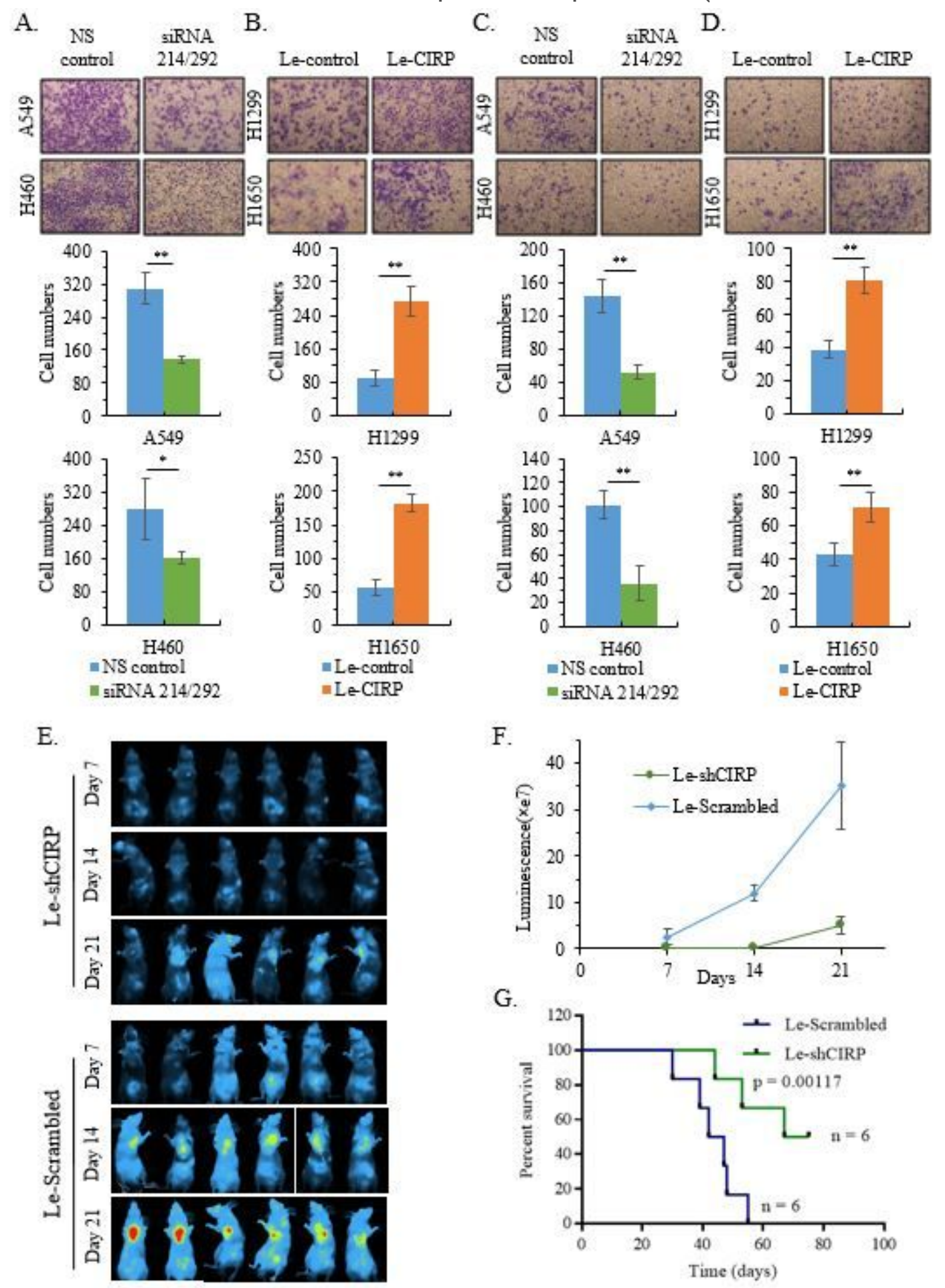


\section{Figure 3}

CIRP Promotes the Aggression of NSCLC Cells. (A) The representative microphotographs showed the migration of A549 and $\mathrm{H} 460$ cells in which CIRP expression was silenced by siRNA. The migrated cell numbers were shown in histograms. (B) The representative microphotographs showed the migration of CIRP overexpressed $\mathrm{H} 1299$ and $\mathrm{H} 1650$ cells. The migrated cell numbers were shown in histograms. (C) The representative microphotographs showed the invasion of A549 and H460 cells in which CIRP expression was silenced by siRNA. The invaded cell numbers were shown in histograms. (D) The representative microphotographs showed the invasion of CIRP overexpressed $\mathrm{H} 1299$ and $\mathrm{H} 1650$ cells. The invaded cell numbers were shown in histograms. All data were means \pm SD from three independent experiments $(* *, P<0.01)$. (E) luciferase images of mice at 7,14 , and 21 days post tail intravenous injected Le-shCIRP or Le-Scrambled infected A549-Luc+ ( $n=6$, per group). (F) The average of bioluminescence signals in Le-shCIRP group at different time points were respectively compared with that in A549-Luc+ cells transduced with Le-Scrambled group (mean $\pm S D ; n=6, * \star, P<0.01$ ). (G) Survival analysis of mice implanted with A549-Luc+ cells with or without CIRP knockdown. 
A.

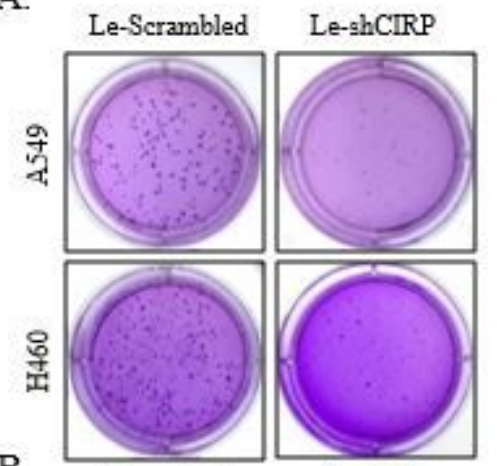

B.
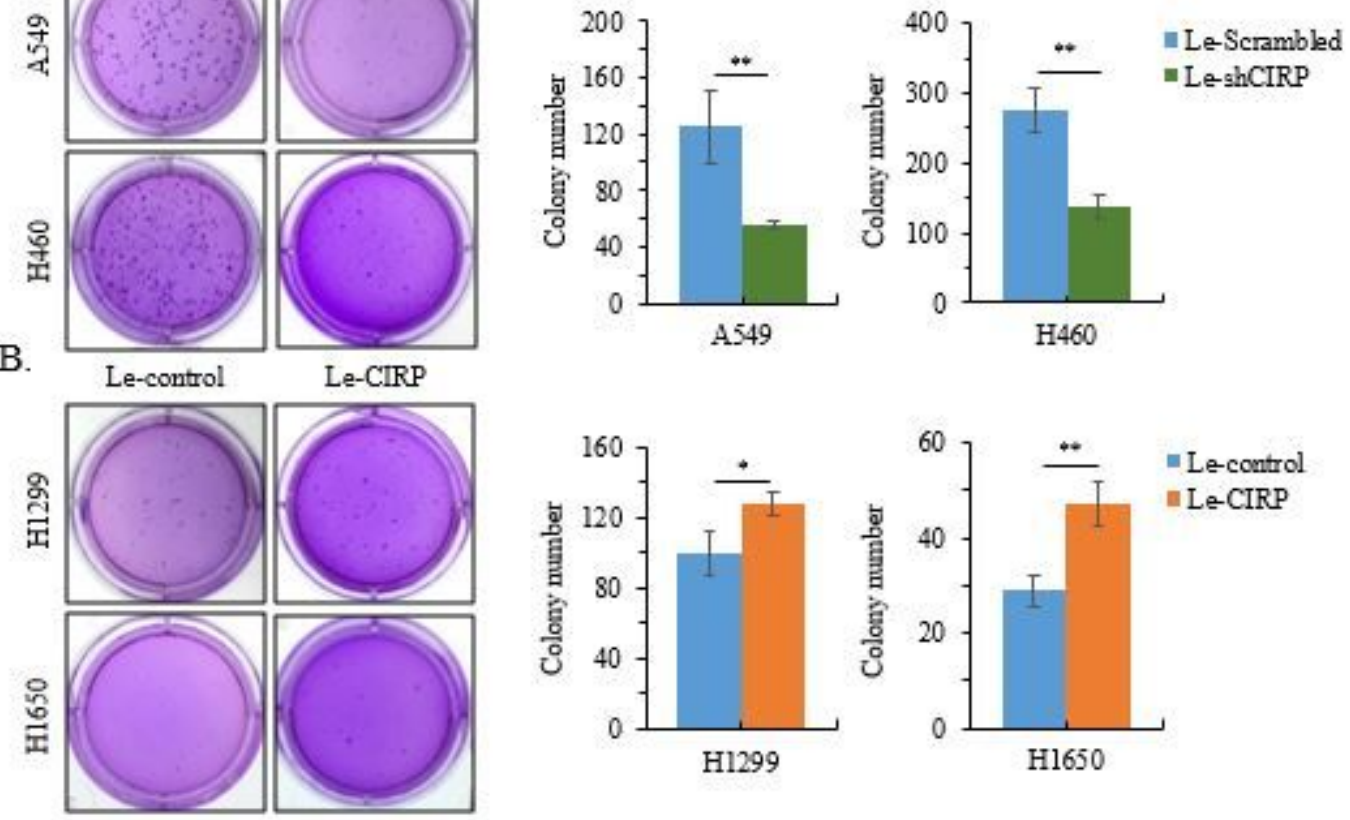

C.

$$
\begin{aligned}
& \begin{array}{l}
\text { Le-scrambled } \\
(\mathrm{n}=9)
\end{array} \\
& \begin{array}{l}
\text { Le-ghCIRP } \\
(\mathrm{n}=9)
\end{array}
\end{aligned}
$$

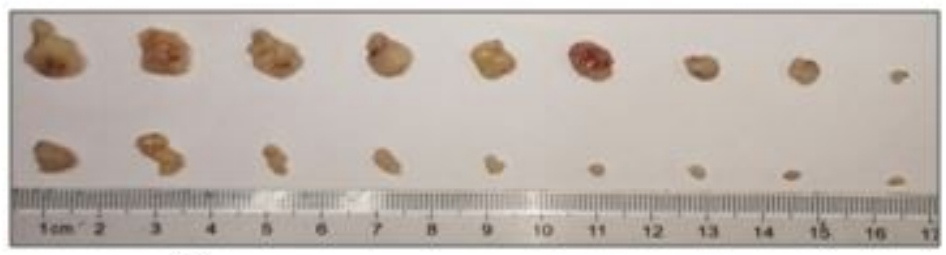

D.

E.
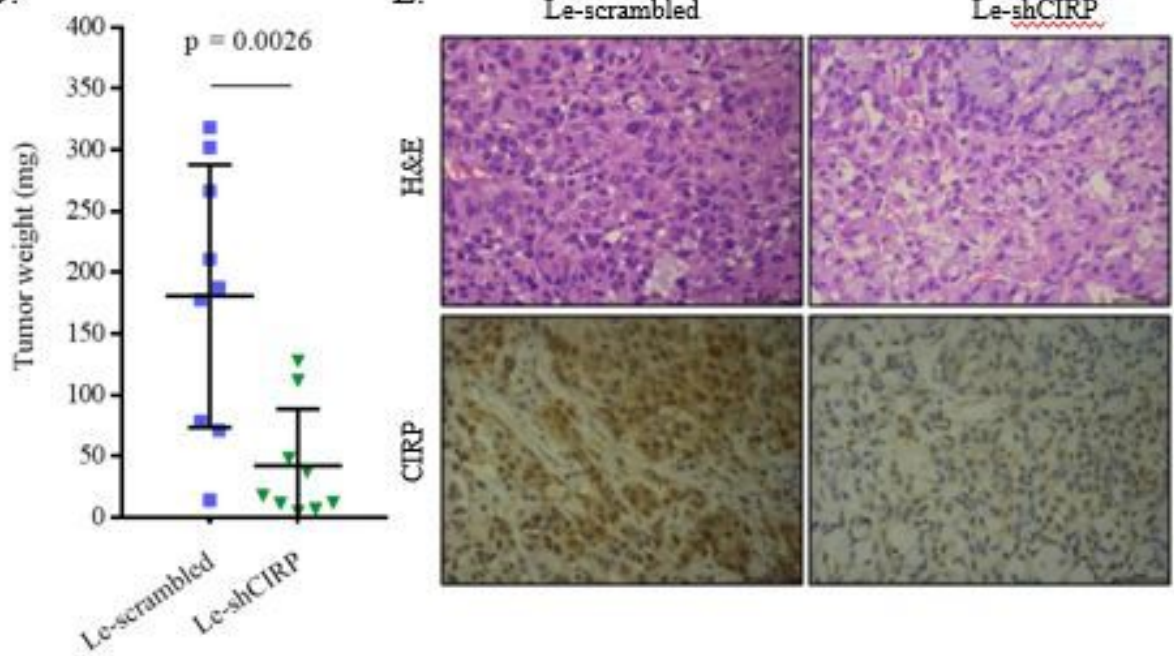

\section{Figure 4}

CIRP Promotes the Tumorigenesis of NSCLC Cells. (A) Representative photographs showed the effect of silencing CIRP on the colony formation of A549 and H460 cells. The clone numbers were shown in histograms representing means \pm SD from three independent experiments $(* \star, P<0.01)$. (B) Representative photographs showed the effect of CIRP overexpression on the colony formation of $\mathrm{H} 1299$ and $\mathrm{H} 1650$ cells. The clone numbers were shown in histograms representing means \pm SD from three independent experiments $(*, P<0.05$ and $* *, P<0.01)$. (C) A549 cells were subcutaneously inoculated in BALB/c nude 
mice after infection with Le-scrambled or Le-shCIRP at MOI of 10 ( $n=9$ for each group). Twenty-eight days later tumors were harvested. (D) The weight of established tumors was measured and was shown in a scatter plot. (E) Immunohistochemical analysis of CIRP expression was performed on these xenografts. Representative images were shown (magnification $\times 200$ ).

A.

TOP 10 Pathway enrichment

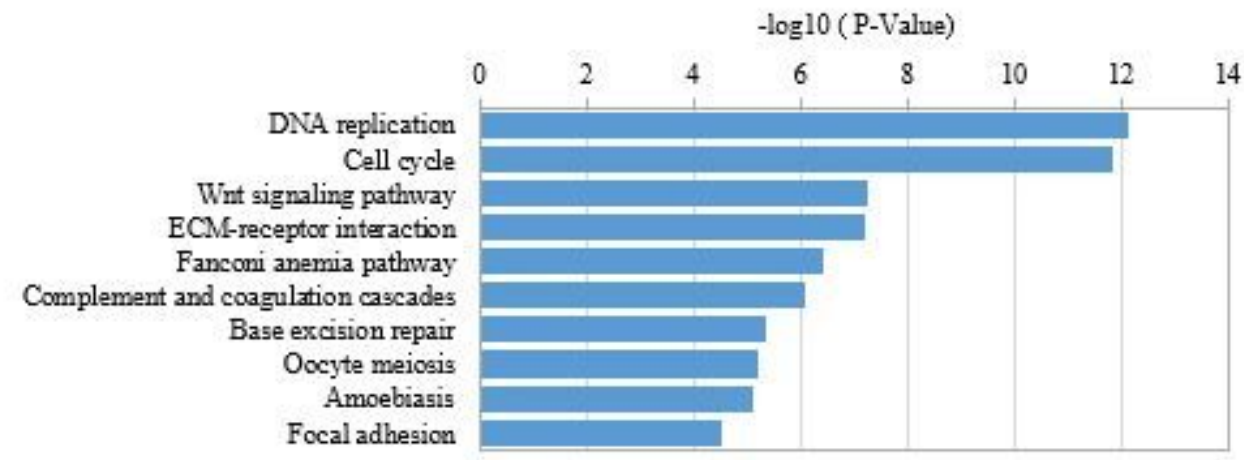

B.

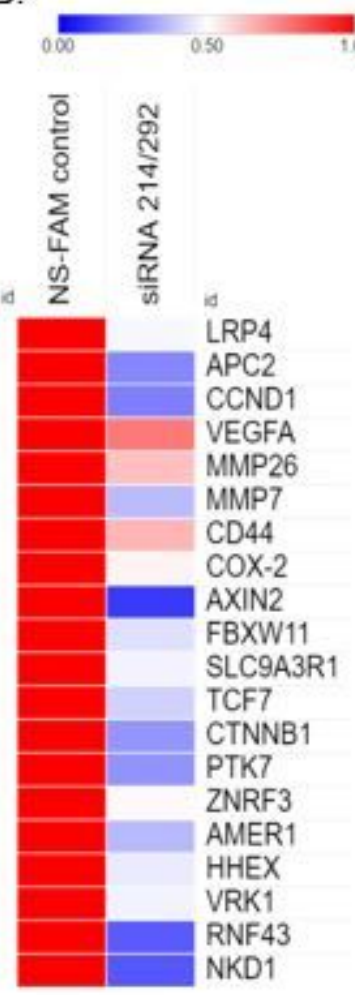

C.

D

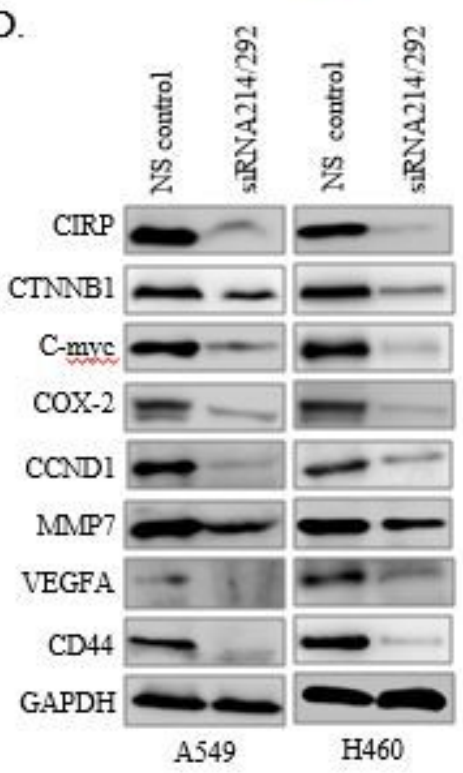

E.

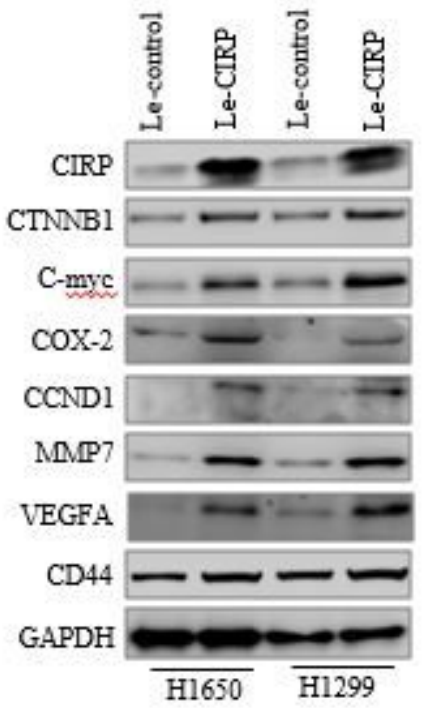

\section{Figure 5}

CIRP Regulates the Wnt/ $\beta$-Catenin Signaling Pathway in NSCLC Cells. (A) The enriched top 10 pathways affected by CIRP downregulation in A549 cells were shown. (B) Heat map showed the mRNA levels of genes involved in Wnt signaling pathway in CIRP silenced A549 cells. Red and blue respectively indicated 
higher and lower gene expression, and the magnitude of expression differences was displayed by color intensity. (C) qRT-PCR analysis the mRNA levels of Wnt/ $\beta$-Catenin signaling pathway related genes was performed in CIRP silenced A549 cells. Data were normalized the levels in NS control A549 cells, and shown as mean \pm SD from three independent experiments. (D) Protein level of indicated genes in CIRP silenced A549 and H460 cells were determined by immunoblotting. (E) Protein level of indicated genes in CIRP overexpressed H1299 and H1650 cells were determined by immunoblotting.

A.

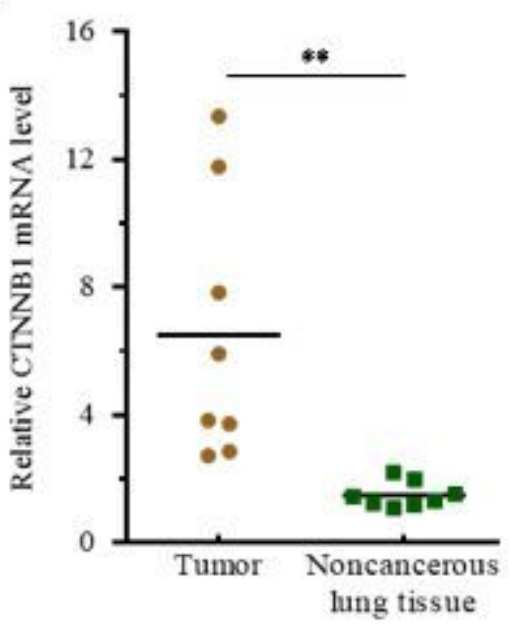

B

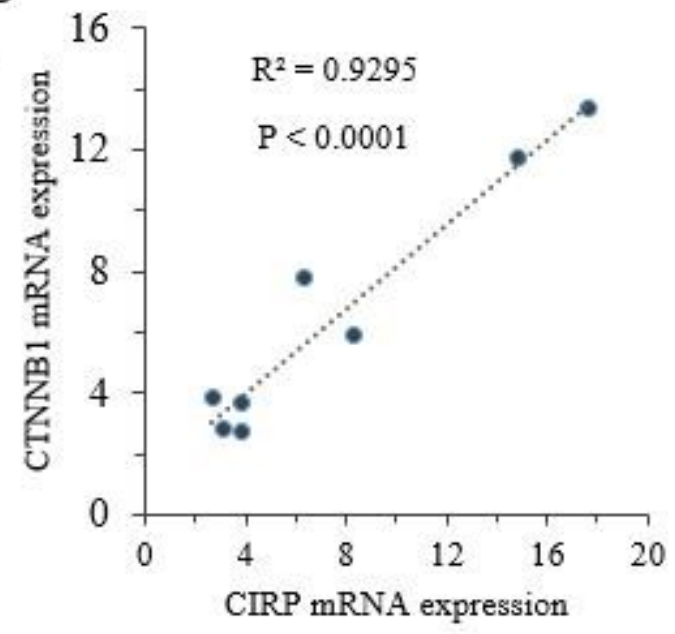

$\mathrm{C}$ Scatter plot-correlation between CIRP and CTNNB1 in lung cancer tissues

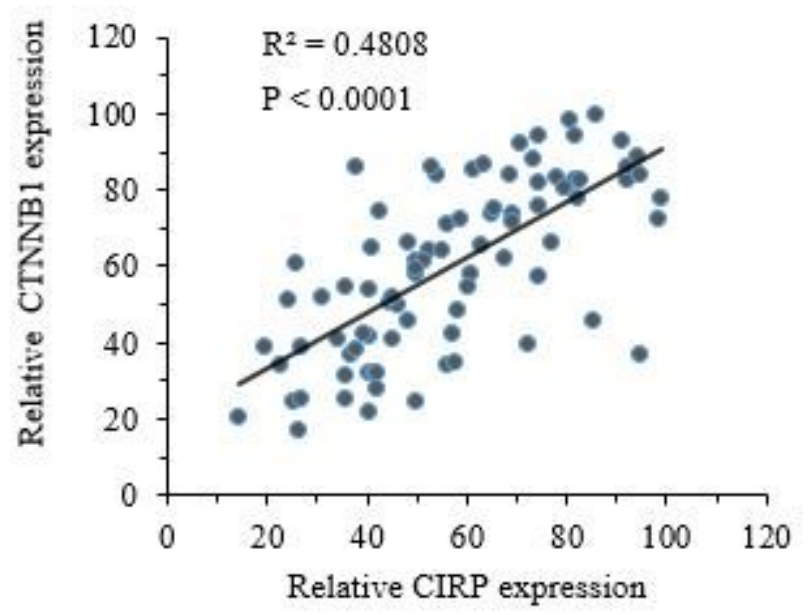

\section{Figure 6}

Expression of CIRP and CTNNB1 is Positive Correlation in NSCLC Tissues. (A) mRNA levels of CTNNB1 were quantified in NSCLC samples and their corresponding noncancerous lung tissues as well as normal lung tissues by qRT-PCR $(n=8)$. CTNNB1 expression was normalized by GAPDH expression and each noncancerous lung tissue was used as a control. Data were shown as mean $\pm S D(\star \star, P<0.01)$. (B) The correlation was shown between CIRP mRNA and CTNNB1 mRNA expression in in NSCLC tissues. (C) The 

plot.

A.

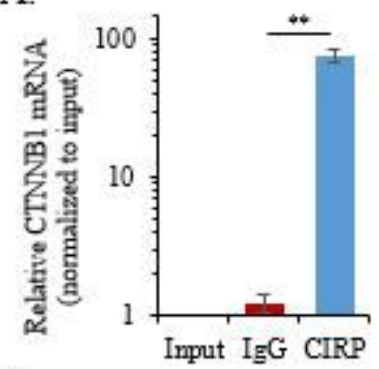

D.

pC3.1/Control report

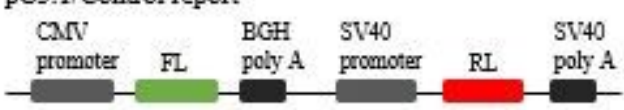

pC3.1/5'-UTR report

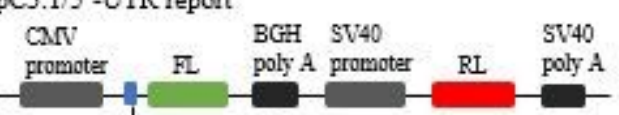

CINB1 5'-URR 214 bp

pC3.1/3'-UTR report

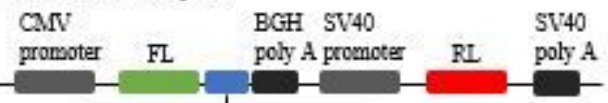

CTNNB1 3'-UIR, 802 bp
B.

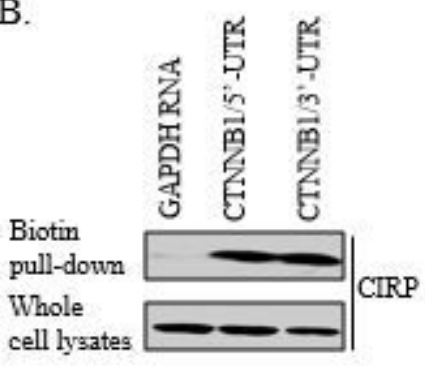

E.

E.

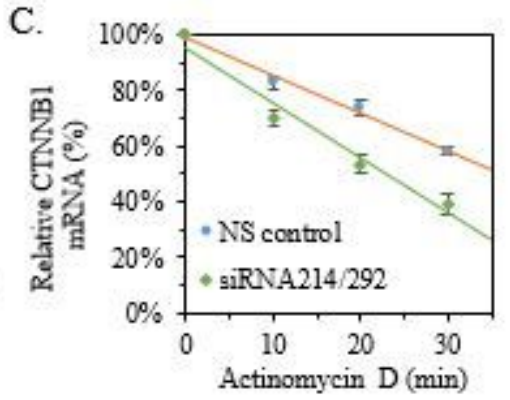

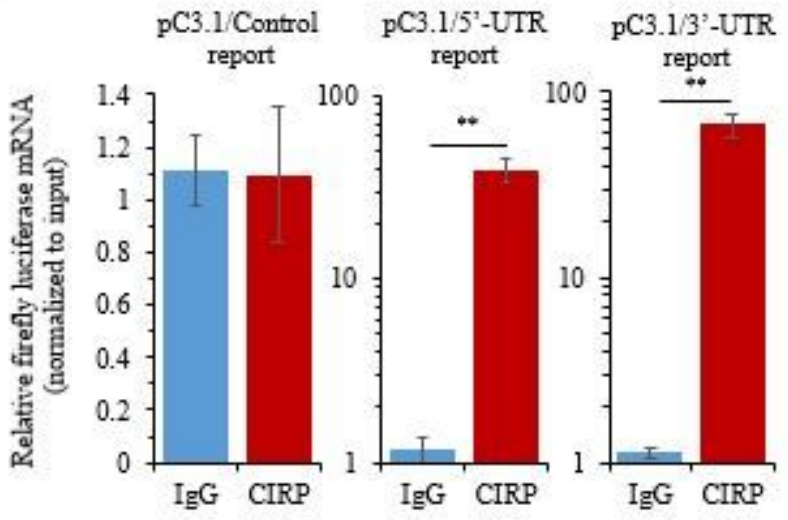

F.

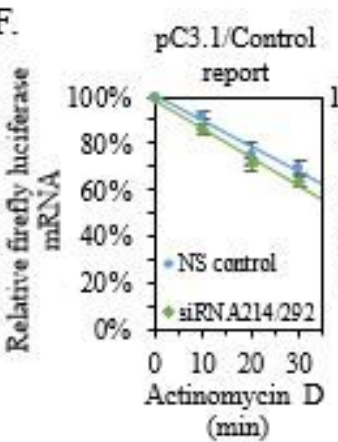

pC3.1/3'-UTR G

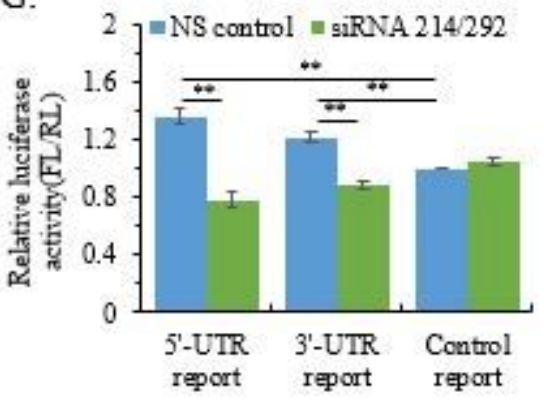

$\mathrm{H}$.

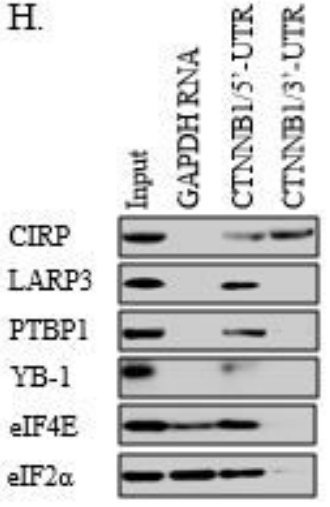

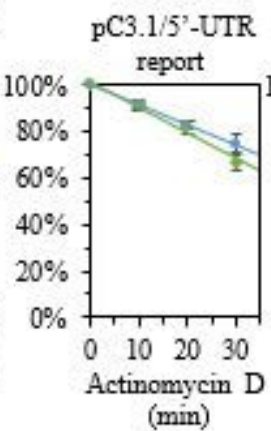

I.

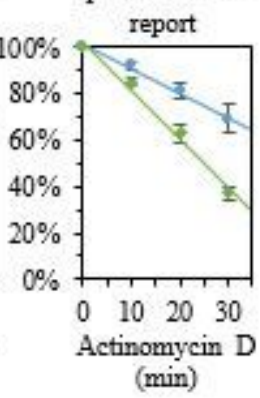

J.

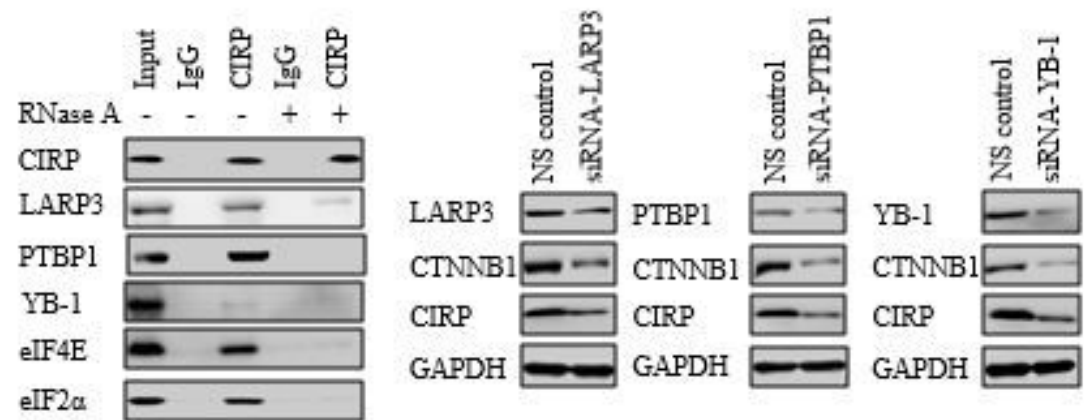

Figure 7

CIRP Post-transcriptional Regulates the expression of CTNNB1. (A) mRNA levels of CTNNB1 in RIP assay were quantified by qRT-PCR. The level in input group was used as control. Data were means \pm SD from three independent experiments ( $*$, $P<0.01)$. (B) Immunoblotting detected the pulled CIRP protein in biotin pull-down assay. Transcript of GAPDH mRNA were used as a negative control. (C) Degradation of 
the CTNNB1 mRNA was analyzed in A549 cells transfected with CIRP or control siRNAs by qRT-PCR. Data were means \pm SD from three independent experiments. (D) The schematic diagrams of indicated luciferase report vectors. (E) mRNA levels of firefly luciferase in RIP assay were quantified by qRT-PCR. Data were normalized to input group and presented as means \pm SD from three independent experiments $(* \star, P<0.01)$. (F) Degradation of the firefly luciferase mRNA were analyzed by qRT-PCR in A549 cells which were respectively co-transfected with luciferase report vectors and CIRP or control siRNAs. Data were shown as means \pm SD from three independent experiments. $(G)$ Luciferase expression was measured in A549 cells which were co-transfected with CIRP or control siRNAs with luciferase vectors. The fold changes of relative luciferase activity in CIRP siRNA with indicated luciferase reporters transfected cells were normalized to NS control with pC3.1/Control report transfected cells. Data were means \pm SD from three independent experiments $(* \star, P<0.01)$. $(H)$ Immunoblotting detected the indicate proteins in biotin pull-down assay. 20 $\mathrm{gg}$ of whole cell lysates of A549 cells (Input) served as a positive control. (I) Immunoblotting analysis of indicated proteins in input and elute upon co-purification with antiCIRP or IgG from A549 cells. Immuno-purifications were performed in the absence (-) or presence (+) of $10 \mathrm{mg} / \mathrm{mL}$ RNase A. (J) Immunoblotting analysis of CIRP and CTNNB1 expression in indicated IRES trans-acting factor silenced A549 cells were performed.

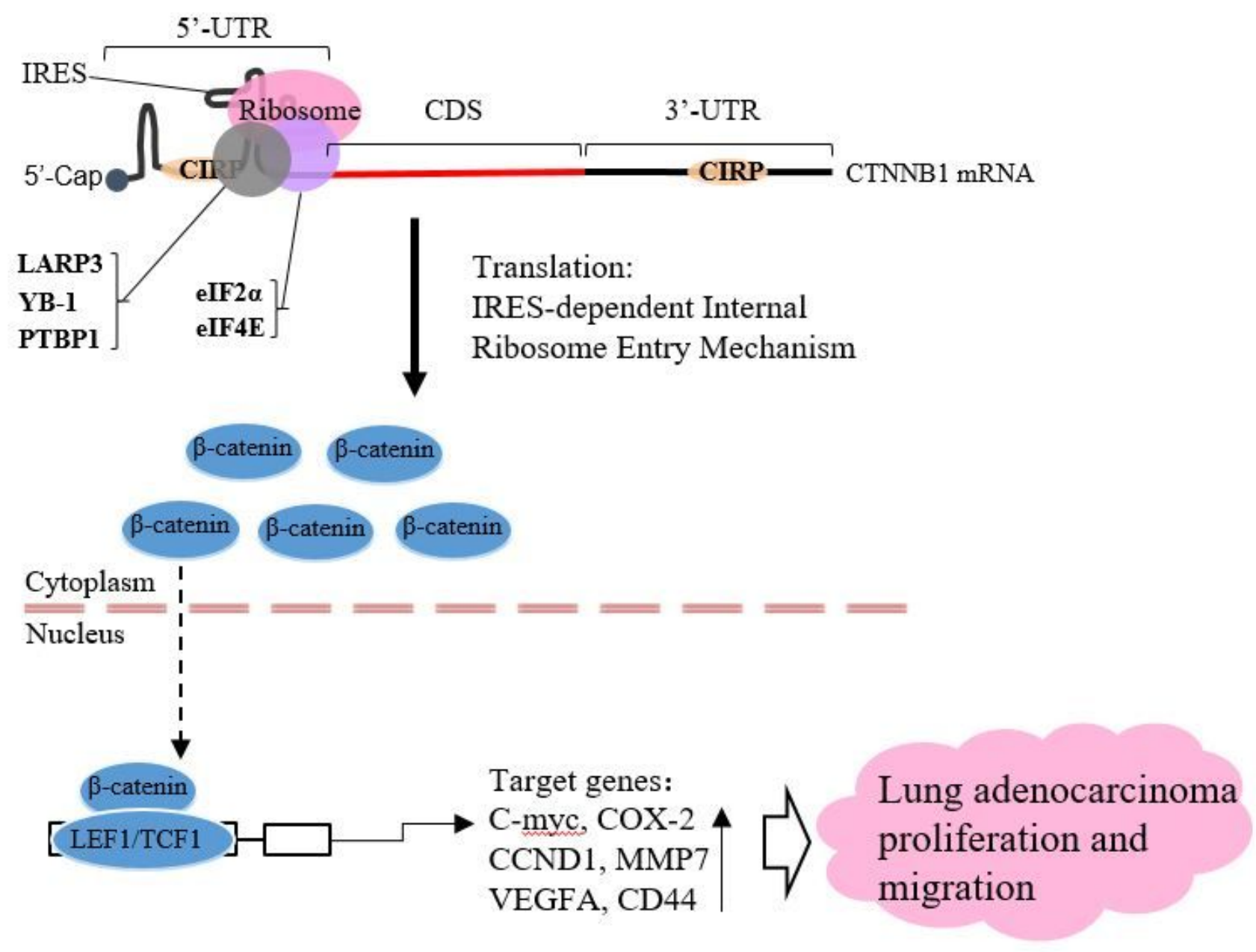




\section{Figure 8}

A mechanism of CIRP-enhanced Wnt/ $\beta$-Catenin Signaling Pathway. The abnormal increased cytoplasm CIRP in NSCLC cells promotes CTNNB1 protein expression through post-transcriptional regulation. That is by binding to the $5^{\prime}$-UTR and $3^{\prime}$-UTR of CTNNB1 mRNA to increase its mRNA stability and facilitate its IRES-dependent translation. The elevated CTNNB1 protein persistently activates the Wnt/ $\beta$-catenin signaling pathway which result in increasing of downstream oncogenes expression and promoting NSCLC progression.

\section{Supplementary Files}

This is a list of supplementary files associated with this preprint. Click to download.

- 4.SupplementaryFigures.pptx

- 5.SupplementaryMaterialsandMethods.docx 